\title{
Diseño de un sistema automatizado para administración de alimento en cultivo de tilapias
}

\author{
Gustavo Delgado Cortez / Pablo Jácome Ruiz
}

\section{RESUMEN}

Esta investigación tiene como objetivo general, diseñar un sistema automatizado que permita la monitorización y control de la administración de alimentos para cultivos de "tilapias". Este sistema representa en un prototipo que utiliza tecnología inalámbrica para la ejecución de la administración de alimentos y un programa tipo scada (Supervisory Control And Data Acquisition) que permite monitorizar los estados de funcionamiento del prototipo en tiempo real. Para operar este sistema solo basta con ejecutar la aplicación y monitorear los estados de cada equipo remoto, que en caso de ser necesaria una modificación se escribirá el comando en la pestaña 'control' del programa. El equipo coordinador de la red inalámbrica, llamado así porque me permite controlar la red de equipos remotos desde el computador con la aplicación de escritorio o scada, se comunica con cada equipo para ordenar el despacho de alimento y su configuración. Además, los equipos remotos basarán su funcionamiento en el uso de un sistema fotovoltaico ya que el acceso con líneas de energía eléctrica convencional a estas áreas en campo abierto es muy difícil y no viable; por otro lado estas energías son amigables al ambiente por no emitir contaminantes a la atmosfera que podría también afectar los cultivos. Las pruebas realizadas al sistema consistieron en comunicar los módulos de forma inalámbrica para la transmisión de instrucciones desde la aplicación de escritorio, el desempeño del equipo remoto según las instrucciones impartidas y la visualización de estados de funcionamiento del equipo remoto en la aplicación de escritorio. Como resultado final se pudo concluir que el sistema superó las pruebas establecidas y cumple con los requerimientos técnicos establecidos, protocolos de comunicación, interfaz hombre máquina y precisión de despacho de alimento, cumpliendo el objetivo de gestionar eficientemente el alimento en cultivos de "tilapias".

\section{PALABRAS CLAVE}

Sistema automatizado, comunicación inalámbrica, equipo coordinador; equipo remoto, sistema scada, microcontroladores, módulo Xbee.

\section{ABSTRACT}

The research goal was to develop an automatic food administration system for a "tilapia's farm. This system is a prototype that monitors and controls several devices using wireless technology. The control functions are for food administration and monitoring functions use scada software in real time for each remote device. If necessary, the operator can use a command to modify the operation of the device in the control tab. The coordinator's device, the one that controls the rest of devices of the red let see status from a computer using the scada and communicates with each remote device to give the food and configure its parameters. 
This system uses solar energy by the photoroltaic system, an energy that cares environment and feeds the system because conventional energy is almost impossible to arrive in those places and it's not riable. Tests applied to the șstem were about the communication of Xbee wireless modules to transmit instructions from the desktop application, the performance of the remote device with the given instructions and the status display of the remote device in the application. $A$ s results of this paper can be concluded that the system exceeded the tests, passes technical recuirements, protocol communications, human machine interface and food administration accuracy fulfilled efficiently manage the food "tilapia" crops.

\section{KEYWORDS}

Automated sy'stem, mireless communniation, coordinator dericu, remote derice, scada s)'stem, miarocontrollers, Noee module. 


\section{Introducción}

\section{Planteamiento del problema}

Las empresas del sector productivo acuicola nacional ecuatoriano, dedicados al cultivo de "tilapias", no utilizan un sistema automatizado para el proceso de alimentación de los cultivos en acuicultura. El método más utilizado es el sistema de "boleo", el cual consiste en que una persona alimenta los cultiros arrojando el alimento a la piscina manualmente (Romo \& Álvarez, 2009).

Ia frecuencia de administración de alimentos que se aplica en la práctica de alimentación es de 2 a 3 reces diarias. (Martinez, (hávez, \& Olvera, 2007). Esto es una limitación del recurso (mano de obra) disponible, cuando se recomienda que la administración del alimento se adapte a las "tilapias", en dependencia de su etapa de desarrollo que puede alcanzar hasta 16 reces diarias (Martíne\%, Chárez, \& (Olvera, 2007).

Además, estudios demuestran que la frecuencia de alimentación es directamente proporcional a la tasa de crecimiento del pe\% (Magdy \& Mohamed, 2008, vol. 20, n³; (howdhury, 2011). Es decir, a mayor cantidad de raciones diarias, mayor tamaño alcanzará el pez.

Se eridencia, por lo tanto, que la alimentación se limita a las posibilidades de la mano de obra y no a las necesidades de la especie. Esto afecta el desarrollo del pez porque no recibe la cantidad de raciones que permita un mayor crecimiento y que deriva en mejor caliclad para la cosecha.

()tros problemas obserrables de la administración manual o no automatizada de alimentos a los cultivos de "tilapias" son:

- limitaciones en la frecuencias de administración de dosis.

- Variabilidad en las dosis con métodos tradicionales
- Falta de monitorización continua del cultivo.

El sistema automatizado se implementa mediante un autómata programable (equipo remoto) ubicado en campo, un equipo controlador central (equipo coordinador) y una red inalámbrica que los comunica. Fil equipo remotos se encargará de proporcionar el alimento al cultivo. Se usa una red inalámbrica para comunicar los autómatas con el controlador central (equipo coordinador). El equipo coordinador procesará las señales y datos provenientes de campo y a su re\% enviará instrucciones hacia los autómatas. Se implementa además, un programa Superivisory, Control And Data Aquisition (SCADA), que permite visualizar los estactos recogidos de campo y registrar datos.

El autómata programable o equipo remoto constará de las siguientes partes: (a) repositorio y base, (b) un sistema fotoroltaico, (c) un microcontrolador, (d) un módulo de comunicación inalámbrica, (e) un servomotor y (f) un sensor ultrasónico.

El repositorio consistiria en envase que contendrá el alimento a proporcionarse al cultivo. Isste repositorio descansará sobre una base que lo mantendrá suspendido por encima del nivel de la piscina.

El sistema fotoroltaico consistiria en tres partes: (a) un panel solar (que captará la radiación solar y transformará la energía solar en energía eléctrica), (b) un regulador de carga (que administrará la energía cléctrica proporcionada por el panel y lo proveerá a las baterias para cargarlas), y (c) baterias (que almacenarán la energía y la proporcionarán al autómata para que este pueda funcionar cuando la energía solar sea nula).

El microcontrolador almacenará el algoritmo de funcionamiento que regirá las ac- 
ciones del autómata programable como: (a) apertura y cierre del servomotor, (b) lectura de datos del sensor ultrasónico, (c) lectura de niveles de voltaje de las baterías y (d) modo de funcionamiento actual.

El módulo de comunicación inalámbrica permitirá el envío y recepción de datos de forma remota desde el equipo remoto y el computador que posee el sistema scada para el control y la monitorización.

El servomotor controlará la apertura y cierre de la compuerta que permitirá la salida del alimento del repositorio hacia la piscina.

El sensor ultrasónico que proveerá la lectura de distancia entre el punto de referencia en el que se ubicará el sensor (en la parte superior del repositorio) y el alimento almacenado en el repositorio. De esta forma se medirá de forma indirecta la cantidad de alimento aun restante en el repositorio, usando la distancia vertical entre el nivel de alimento $\mathrm{y}$ la referencia.

Por último, el sistema scada permitirá visualizar los estados de los autómatas programables ubicados en las piscinas en todo momento, además de poder modificar sus estados remotamente desde el computador.

Este sistema automatizado será capaz de administrar dosis de alimentos previamente programadas y con la frecuencia que el cultivo requiera. Además se podrá controlar remotamente desde una aplicación de escritorio y reprogramar los equipos en campo a distancia.

Los objetivos de este trabajo de titulación son:

- Determinar los requerimientos técnicos de funcionamiento del sistema automatizado.

- Proponer un diseño de sistema au- tomatizado de control de administración de alimento para cultivos de "tilapias".

- Evaluar los parámetros que prueben el correcto desempeño del sistema.

\section{Fundamentación Teórica}

\section{Comunicaciones}

La comunicación de ideas es esencial para que una sociedad funcione ! se desartolle (Sheikh, 2004). Existen tres clases de comunicación: (a) la interactiva; (b) la almacenada; $y$ (c) la difundida.

La comunicación interactiva se define por la transmisión de datos o mensajes, que han sido influenciados por la recepción de mensajes o datos previos; o predice la naturaleza de mensajes futuros.

En automatización, el envío y recepción de datos es común para mantener una monitorización constante de los procesos, concordando de esta forma con Serna (2009) en que el control y monitoreo inalámbrico es una aplicación en auge en el ámbito industrial. Por esta razón, es necesario aplicar tecnologías que permiten la comunicación entre los equipos desde los dispositivos en campo hasta el dispositivo administrador de red que controla y supervisa el correcto funcionamiento del proceso.

Para el desartollo de esta investigación, se simulará un equipo remoto que estaría en campo abierto, el cual administrará alimentos al cultivo. Estos dispositivos enviarán y recibirán datos desde un controlador central encargado de supervisar y gestionar que el proceso se cumpla. La comunicación de esta red de equipos será tanto interactiva (cuando los dispositivos envíen y reciban instrucciones entre sí para actuar inmediatamente), almacenada (los datos almacenados en el microcontrolador) y difundida (cuando el equipo administrador envie instrucciones a 
todos los equipos remotos en campo).

\section{Comunicaciones inalámbricas}

La comunicación inalámbrica es una de las más grandes historias de éxitos de la ingeniería en los últimos 25 años, no solo desde el punto de vista científico, sino en términos del tamaño de mercado y el impacto en la sociedad (Molish, 2011).

El término inalámbrico o vireless, es usualmente utilizado para describir tecnologías que emplean el espacio como medio de propagación y que no está conectado por cables (Singal, 2010).

Para la comunicación de los equipos en la red que controlará el proceso de alimentación de los cultivos de "tilapias", se empleará una red inalámbrica (nireless) ya que el sistema automatizado se encontrará en campo abierto, donde líneas de energía y de datos no tienen facilidad de instalación.

\section{El estándar IEEE 802.15.4}

La tecnología inalámbrica descrita en el estándar 802.15.4 del Institute of Electrical and Electronics Engineers (IEEE) es un sistema de comunicación de corto alcance que proporciona aplicaciones de bajo rendimiento y latencia en redes personales inalámbricas Wireless Personal Area Netmork (WPIN) (Buratti, Martaló, Verdone, \& Ferrari, 2011).

Este estándar define el nivel físico y de control de entrada al medio de las redes WPAN con el fin habilitar la comunicación entre módulos con baja tasa de transmisión de datos.

\section{Zigbee}

"Zigbee es una tecnología inalámbrica de corto alcance, bajo consumo de potencia, baja transmisión de datos y alta seguridad de uso creciente en diversas aplicaciones."'(Serna, 2009)
De acuerdo a Farahini (2008), ZigBee es un estándar que define un conjunto de protocolos de comunicación wireless de baja tasa datos de corto alcance.

ZigBee es una tecnología wireless que se desarrolló sobre una estándar global abierto para direccionar las necesidades únicas como bajo costo y bajo consumo de las redes wireless Macbine to Machine (Digi, 2013). Esta tecnología trabaja bajo las especificaciones físicas de radio del IFEIE 802.15.4 y opera dentro de las bandas no licenciadas.

Las características de este protocolo son:

- Soporta múltiples redes topológicas tales como: (a) punto a punto, (b) punto a multipunto y (c) malla.

- Hasta 65 mil nodos por red.

- Erasión de colisiones, reintentos y reconocimientos.

En la tecnología Zighee se reconocen tres tipos de dispositivos: (a) el ZigBee Coordinator (ZC), (b) ZigBee Routery (c) ZigBee Find Derice. $\mathrm{I}: \mathrm{ZC}$ es aquel que administra la red y es el único que puede iniciar la red. I.uego, este ZC será el que acepte que algún '/igBee Ronter () alyún ZigBee Iind Derice (ZISD) se una a la red para que la red tenga un mayor alcance (Chia-Ning, Ruay-Shiung, Pu-I, \& Jei-Hsiang, 2011).

Los equipos remotos distribuidos en campo, enviarán datos capturados por sensores, en este caso la red recibe el nombre de WSN (Wireless Sensors Network). Ista red está implícita en la red ZigBee ya cue Budjei y Moraru (2011), describen también tres tipos lógicos de dispositivos de red o nodos: (a) coordinator (solo existe uno en la red y es el que inicia la red), (b) router (pueden existir tantos nodos en la red como se desee para incrementar el área de transmisión de clatos inalámbricos) y (c) end node (utilizados para recoger datos del ambiente). 
Para el establecimiento de la red inalámbrica de comunicación para los dispositivos de campo y el dispositivo coordinador central se implementará una red ZigBee, que contará con un coordinator (que será el equipo coordinador y supervisor de la red) y varios routers (que serán los dispositivos dispersos en campo).

\section{Tipos de topologías ZigBee}

De acuerdo a Gislason (2008), las topologías que se pueden manejar en una red ZigBee son: (a) Estrella, (b) Árbol ! (c) Mesh 0 malla.

En la topología estrella, el coordinador se encarga de controlar y supervisar a los end devices que tenga a su cargo. En esta topología no usa routers, cada uno de los dispositivos que recogen datos del ambiente envía directamente al coordinador sin ningún intermediario.

En la topología árbol, el coordinador dispone de routers y puede armar pequeñas subtopologías en estrella y los datos viajan de forma jerárquica hacia el coordinador de la red.

En la topología malla, el coordinador y los routers trazan las rutas más cortas para enviar los datos al destinatario. En esta topología, los equipos se encuentran enlazados entre sí, a diferencia de la topología árbol, un end device es capaz de enviar los datos directamente al coordinador o por algún otro router que no esté directamente en su jerarquía.

Para la implementación de la red, se empleará una topología mesh (malla), ya que se considera que es óptima para prever infortunios si algún equipo deja de funcionar, no se corte toda una línea de transmisión, sino que los datos puedan tomar vías alternas.

\section{Scada}

Para Lakhoua y Laadhari (2013), SCADA se refiere a un sistema que colecta datos provenientes de diferentes sensores de un proceso industrial, estos datos son procesados por una estación de procesamiento y sus resultados son enviados a una interfaz humano-máquina como un computador.

Ejemplo de un sistema scada para monitorización en tiempo real es el realizado por Lakhoua y Laadhari (2013), en el que implementaron este sistema para supervisar cuartos de control en una estación cle gas natural. Ellos diseñaron el sistema scada en lenguaje de programación C\#.

Otro ejemplo similar es el scada diseñado en la investigación de Serna (2009), en el que propuso un sistema scada que monitorice señales de sensores montados bajo una red ZigBee como la que se propone en esta investigación. Serna basó su scada en el programa LabView.

Para esta investigación se propone desarrollar el sistema scada para la monitorización y control de estados en Visual Studio. A diferencia de los trabajos citados, este sistema no solo monitoreará estados, sino que también podrá modificar el funcionamiento actual de los equipos en red.

\section{Metodología}

\section{Diseño de la investigación}

El diseño de investigación de este trabajo de titulación será de tipo exploratoria experimental y documental, debido a que la información será obtenida mediante la revisión de libros y revistas científicas, referentes a las diferentes tecnologías que involucra el prototipo a diseñar. También se ejecutan experimentos controlados que permiten determinar la validez de los diferentes bloques de diseño que se proponen en el presente trabajo de investigación. 
Para la evaluación del sistema se procederá con un proceso de observación del funcionamiento general del sistema. Para ello es necesario ubicar los elementos más importantes que componen el sistema automatizado, como se muestra en la Figura 1 y en la Figura 2.

proporcione, girando hacia la izquierda o derecha mediante el uso del bloque Pulse Width Modulation (PWM) del microcontrolador.

En segundo lugar, se procederá con las pruebas de funcionamiento del sensor ultrasónico, midiendo los niveles de voltaje que el sensor arroje para de-

\section{Figura \# 1}

\section{Arquitectura de red del sistema.}

Muestra los elementos más importantes que componen tanto al equipo remoto (a la izquierda del gráfico) y al equipo coordinador (a la derecha del gráfico).

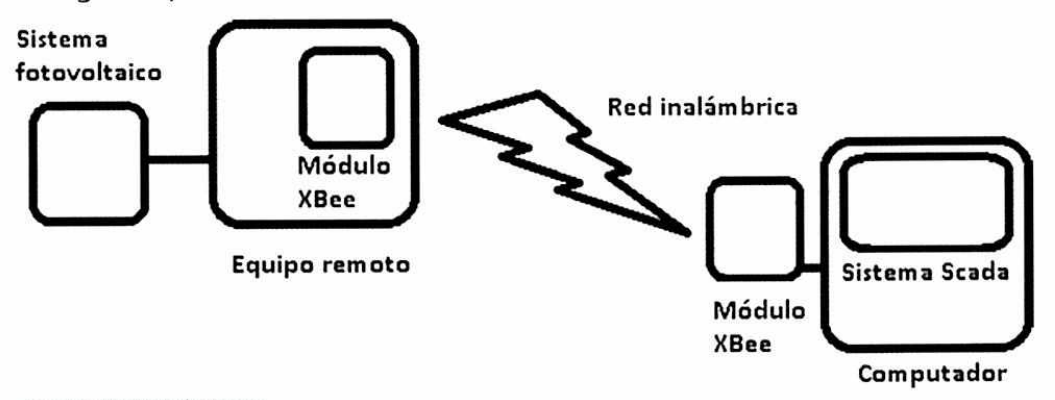

Fuente: Elaboración propia

Figura \#2

Diagrama de bloques del equipo remoto.

Muestra en detalles los elementos que conforman el equipo remoto del sistema.

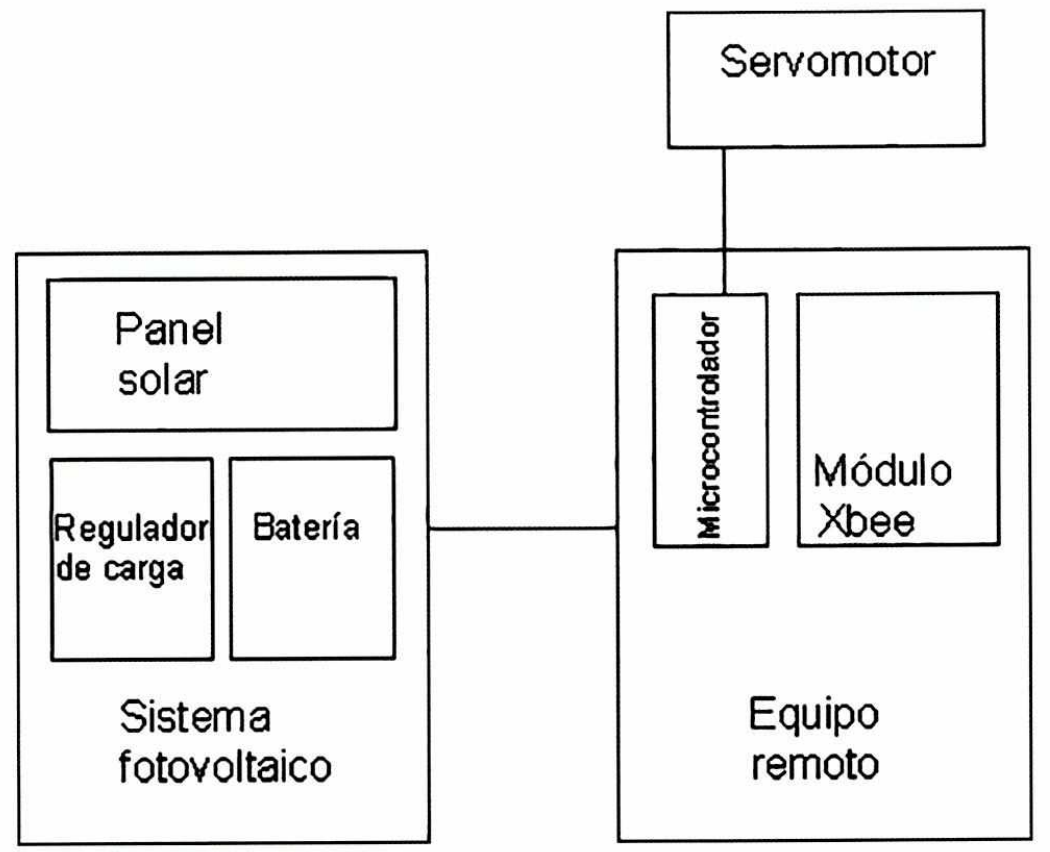

Fuente Elaboración propia

En primer lugar, se procederá con las pruebas del servomotor, el cual habilitará el paso del alimento a la piscina. El motor actúa en dependencia del nivel de voltaje que se le
Computador

terminar distancias. Se comprobará el correcto funcionamiento del sensor mediante la medición de las distancias entre un objeto y el sensor en contraste con los valores que este último determine.

\section{Programadas las} funciones, se dará paso a la elaboración del circuito en protoboard

Después, se configurarán los parámetros de comunicación de los módulos inalámbricos Xbee para establecer la red de comunicación transparente entre los módulos, esto es, que los datos emitidos desde uno de estos módulos, serán visibles en todos los equipos de la red en tiempo real. Para la transmisión de información se establecerá un protocolo de trama de datos para identificar los comandos que viajan por la red inalámbrica.

Luego, se programará la aplicación de monitorización y control de la red. Una vez hecho esto, se simulará el envío de datos al coordinador de la red para que estos datos sean visibles en la aplicación y poder así apreciar el co- 
rrecto funcionamiento de la comunicación entre el microcontrolador del coordinador de la red y el sistema Scada.

\section{Requerimientos técnicos}

Como se demostró en la introducción, el manejo de dosis y frecuencias de alimentación es el punto central que debe poder manejarse sin que dependa del ser humano, por ello se debe establecer una rutina que permita alimentar el cultivo manipulando estas dos variables.

Este sistema tendrá 4 estados posibles tanto para frecuencia como en dosis de alimentación. Para el desarrollo del programa que controlará al sistema, se establecerán los estados para frecuencia de alimentación: (a) 10, (b) 15, (c) $30 \mathrm{y}$ (d) 45 minutos. Para dosis de alimentación se programarán (a) 100 , (b) 200 , (c) $300 \mathrm{y}$ (d) 400 gramos de alimento para tilapia.

Para el sistema scada, se necesitará monitorear estas variables para poder determinar bajo qué estado se encuentran funcionando actualmente, dando posibilidad a que se puedan modificar.

Además, también será necesario monitorear estados actuales de nivel de batería y de alimento. Para lograr esto, se programará un sistema de alarmas de nivel para estas dos variables que debe indicar: (a) nivel alto, (b) nivel medio y (c) nivel bajo.

Por último, deberá tener la posibilidad de modificar sus estados de funcionamiento de forma local, es decir, cuando un operador se encuentre en el panel de control del equipo remoto. Esto consistirá en tres botones: (a) para cambiar de modo automático a modo manual, (b) un botón para modificar la frecuencia de administración de alimento y (c) un botón para modificar la dosis. El primer botón, al ser presionado cambiará el estado actual del sistema (si se encuentra en manual, los botones de frecuencia y dosis serán habilitados para modificar los estados, caso contrario estos estará inhabilitados).

\section{Requerimientos energéticos}

Los consumos de energía de cada dispositivo que compone el circuito se detalla en la Tabla 1.

\begin{tabular}{|c|c|c|c|}
\hline \multicolumn{4}{|c|}{$\begin{array}{l}\text { Datos de voltaje, corriente y potencia de los elementos } \\
\text { electrónicos utilizados en el equipo remoto. Los datos } \\
\text { mostrados indican el consumo de cada elemento de } \\
\text { forma continua. }\end{array}$} \\
\hline Dispositivo & Voltaje (V) & Corriente $(A)$ & Potencia (W) \\
\hline HC-SRO4 & 5 & 0,015 & 0,075 \\
\hline $\begin{array}{c}\text { Microcontrolador } \\
\text { PIC 16F887 }\end{array}$ & 5 & 0,00055 & 0,00275 \\
\hline $\begin{array}{l}\text { Microcontrolador } \\
\text { PIC 16F886 }\end{array}$ & 5 & 0,00055 & 0,00275 \\
\hline Módulo Xbee & 3,3 & 0,04 & 0,132 \\
\hline Servomotor & 5 & 0,3 & 1,5 \\
\hline Consumo & $\cdots$ & 0,3561 & 1,7125 \\
\hline
\end{tabular}

El fin del desarrollo del sistema es que este funcione las 24 horas del día. La tabla anterior nos muestra el consumo por hora de funcionamiento de cada elemento del equipo remoto, pero se debe determinar el consumo por hora del servomotor ya que este no funciona de forma continua.

Como máximo se estima que el servomotor que habilita el paso del alimento al cultivo funcione durante 1 minuto por cada hora.

Con este valor, los amperios por hora que consumiría cada elemento del equipo remoto se muestra en la siguiente Tabla 2. 


\section{Tabla \#2}

\section{Consumo de corriente de cada elemento del equipo remoto en unidades Amperios/hora.}

\begin{tabular}{cc} 
Dispositivo & Corriente $(\mathrm{A} / \mathrm{h})$ \\
HC-SR04 & 0,015 \\
\hline PIC 16F887 & 0,00055 \\
\hline PIC 16F886 & 0,00055 \\
\hline Módulo Xbee & 0,04 \\
\hline Servomotor & 0,005 \\
\hline Consumo & $\mathbf{0 , 0 6 1 1}$ \\
\hline
\end{tabular}

Valores obtenidos de las hojas de datos (datasheets) de los dispositivos Fuente: Elaboración propia

Obtenido el consumo real por hora del equipo remoto, se debe multiplicar este valor por la cantidad de horas que estará funcionando al día.

$$
\begin{gathered}
\text { Amperios bora estimados por consumir } \\
(0.0611 \mathrm{~A})(24 \mathrm{~h})=1.47 \mathrm{~A} / \mathrm{h}
\end{gathered}
$$

Para alimentar el equipo remoto se plantea usar baterías de $12 \mathrm{v}$ a $4 \mathrm{~A} / \mathrm{h}$, pero para determinar la cantidad de baterías de esta capacidad necesarias se recurrió al siguiente cálculo:

Número de baterías $=\frac{2(1.47 A / h)}{4 A / h}=0.73$

Con este resultado, se puede afirmar que bastaría 1 batería de $12 \mathrm{v}$ a $4 \mathrm{~A} / \mathrm{h}$ para alimentar el equipo remoto.

Como los requerimientos energéticos de los elementos del sistema indican una tensión de $5 \mathrm{vdc}$, se contempla colocar un regulador de voltaje LM338 para disminuir la tensión de la batería y proporcionar el requerido por el sistema.

Para poder cargar las baterías se optó por el uso de un sistema fotovoltaico ya que el equipo remoto se encontraría aislado a campo abierto, donde no es posible alimentar el sistema con energía eléctrica convencional. El sistema fotovoltaico que requeriría el equipo remoto es: (a) un panel solar y (b) un regulador de carga.
El panel solar captaría la radiación solar y transformaría la energía solar en energía eléctrica; el regulador de carga administrará la energía eléctrica obtenida por el panel solar para cargar las baterías.

La capacidad del sistema fotovoltaico que puede mantener el funcionamiento continuo del equipo remoto es $5 \mathrm{~W}$ de acuerdo al siguiente cálculo.

\section{Corriente del sistema fotovoltaico}

$$
\frac{5 W}{12 V}=0.5 \mathrm{~A}
$$

Este sistema fotovoltaico proporciona $500 \mathrm{~mA}$, una corriente 8 veces mayor a la de consumo del sistema $(61 \mathrm{~mA})$.

\section{Protocolo de comunicación}

El protocolo de comunicación para el envío de tramas de datos desde el coordinador hacia el equipo remoto que se desarrolló consta de 8 caracteres como se describe en la Figura 3.

\section{Figura \#3}

Estructura de trama de datos

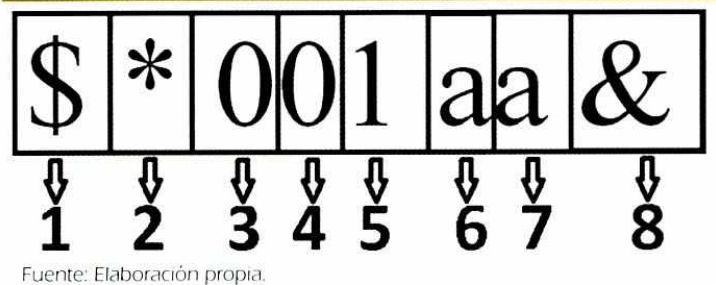

Los primeros 2 caracteres (1) \$y (2) * tienen fin de distinguir la cabecera de la trama. En la rutina del microcontrolador del equipo remoto, cuando se identifica en la trama el símbolo " $\$$ " se borra cualquier valor que se encuentre en las casillas de la variable que almacena la trama de datos que se recibe.

Las siguientes tres casillas (3) 0 , (4) $0 \mathrm{y}$ (5)1 identifican al equipo remoto al que la trama fue dirigida; en este caso el equipo remoto es el denominado "001". La rutina del microcontrolador, luego de identificar la ca- 
becera procede a evaluar estos 3 caracteres para poder distinguir si la trama recibida fue dirigida específicamente para él y de esta forma responder al equipo coordinador sobre aquel dato requerido.

En una red tipo malla de comunicación transparente, como la diseñada para este sistema, las tramas enviadas desde el equipo coordinador son recibidas por todos los nodos terminales que se encuentran en la red.

Las casillas (6) "a" y (7) "a" corresponden al dato que requiere el equipo coordinador del equipo remoto. La casilla (6) corresponde al tipo de instrucción que envía el equipo coordinador para un determinado equipo remoto: la letra "a" indica modificación, y la letra "b" indica requerimiento de dato. $\mathrm{La}$ casilla (7) tiene cuatro variables:
(a) dosis
(b) frecuencia
(c) nivel de alimento
(d) nivel de batería
(e) modo de funcionamiento (si está en manual o en automático)

Entonces, la combinación de letras posible con las casillas 6 y 7 es la siguiente Tabla 3:

\section{Tabla \#3}

\section{Combinación de letras entre casillas 6 y 7 de la trama de datos.

Dato Casilla 6 Casilla 7 Mensajes

\begin{tabular}{cccc}
1 & a & a & Modificar dosis \\
\hline 2 & a & b & Modificar frecuencia \\
\hline 3 & b & a & Requerimiento de dato de dosis \\
\hline 4 & b & b & Requerimiento de dato de frecuencia \\
\hline 5 & b & c & Requerimiento de dato de nivel de alimento \\
\hline 6 & b & d & Requerimiento de dato de nivel de bateria \\
\hline 7 & b & e & Requerimiento de dato de modo de funcionamiento
\end{tabular}

Fuente Elaboraciòn propia

La instrucción "Modificar dosis" permite cambiar la cantidad de alimento que se proporciona al cultivo. La rutina detecta que cantidad es la deseada contando el número de veces que se recibe la instrucción por par- te del equipo remoto. El número de veces asciende hasta cuatro, es decir, si el del valor del contador es 1, el tiempo que el servomotor (que controla la compuerta de salida de alimento) mantiene es 2 segundos; si el contador es 2, el tiempo de acción del servomotor es de 4 segundos; si el contador es 3 , el tiempo será de 5 ; y si el contador es 4 , el tiempo es de 6 . Una vez que el contador llega a 4, el siguiente valor será 1 .

La instrucción "Modificar frecuencia" permite variar el tiempo de espera entre dosis proporcionadas. Al igual que la instrucción "Modificar dosis", en dependencia del valor que registre el contador para la frecuencia de administración de dosis variará el tiempo que se cuenta entre raciones proporcionadas en 4 valores diferentes.

Las instrucciones de requerimiento tales como (a) Requerimiento de dato de dosis, (b) Requerimiento de dato de frecuencia, (c) Requerimiento de dato de nivel de alimento, (d) Requerimiento de dato de nivel de batería y (e) Requerimiento de dato de modo de funcionamiento, producen que aquel equipo remoto que haya recibido el requerimiento responda con una trama al equipo coordinador por aquella petición, específicamente con el valor del contador que maneja la instrucción.

En el caso del requerimiento de dato de estado de funcionamiento, la respuesta por parte del equipo remoto es si se encuentra funcionando en modo automático (cuando el equipo remoto es comandado desde el equipo coordinador) o si está funcionando en modo manual (cuando el equipo remoto es comandado localmente desde campo por parte de un operador). 
Técnicas de investigación y pasos a utilizar

Las referencias bibliográficas consultadas fueron pertinentes a: (a) microcontroladores, enfocado a las funcionalidades de los microcontroladores en general y (b) las comunicaciones, orientadas a las comunicaciones inalámbricas como tecnología para el envío y recepción de datos en una red tipo malla.

\section{Tabla de pruebas}

Las pruebas a las que el sistema será sometido se resumen en la siguiente tabla indicando el resultado deseado.

\section{Sistema Scada}

El sistema de comando y visualización de estados consta de dos pantallas: (a) la pantalla de envío y recepción de comandos y (b) pantalla de visualización de estados del sistema.
La pantalla de envío y recepción de comandos permite la escritura de instrucciones desde el equipo coordinador hacia el equipo remoto (véase figura 4), y la recepción de tramas de respuesta desde el equipo remoto hacia el equipo coordinador (véase figura 5).

\section{Figura \#4}

Pantalla de comando del sistema scada.

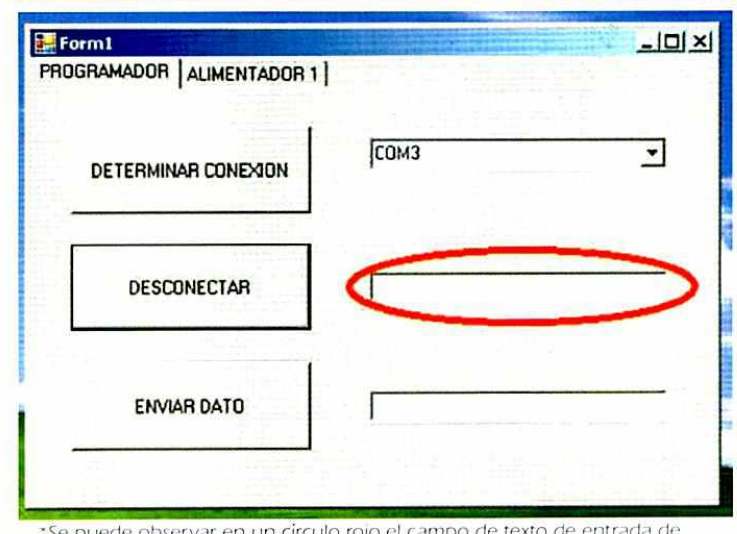

- Se puede observar en un circulo rojo el campo de texto de entrada de comandos.

\section{Tabla \#4}

Pruebas aplicadas al sistema con los resultados que son esperados de la aplicación de las pruebas.

\section{Prueba}

Transmisión de comando: Modificar dosis

Transmisión de comando: Modificar frecuencia

Transmisión de comando:

Requerimiento de dato de dosis

Transmisión de comando:

Requerimiento de dato de frecuencia

Transmisión de comando:

Requerimiento de dato de nivel de alimento

Transmisión de comando:

Requerimiento de dato de nivel de batería

Transmisión de comando:

Requerimiento de dato de modo de funcionamiento

Funcionamiento ciclico del sistema remoto.

Visualización de estados en sistema scada.

Pruebas de comunicación a distancia 50m con linea de vista.

Pruebas de comunicación a distancia $110 \mathrm{~m}$ con línea de vista entre equipo coordinador y equipo remoto.

Pruebas de comunicación a distancia $110 \mathrm{~m}$ con linea de vista entre equipo coordinador y equipo remoto, más un módulo Xbee entre estos dos remoto,

\section{Resultados deseados}

Recepción de la respuesta programada en campo de texto del sistema scada: "100gr", "200gr", "300gr", o "400gr".

Recepción de la respuesta programada en campo de texto del sistema scada: "10min", "15 min", "30min", o "45min".

Recepción de la respuesta programada en campo de texto del sistema scada: "100gr", "200gr", "300gr", o "400gr".

Recepción de la respuesta programada en campo de texto del sistema scada: "10min", "15min", "30min", o "45min".

Recepción de la respuesta programada en campo de texto del sistema scada: "nivelalto", "nivelmedio", o "nivelbajo".

Recepción de la respuesta programada en campo de texto del sistema scada: "nivelalto", "nivelmedio", o "nivelbajo".

Recepción de la respuesta programada en campo de texto del sistema scada: "manual" o "automático".

Equipo remoto complete la rutina programada del accionamiento del servomotor durante por lapso de 1 hora.

Sistema scada muestre los estados de funcionamiento del equipo remoto.

Comunicación normal entre equipo coordinador y equipo remoto.

Comunicación fallida

Comunicación normal entre equipo coordinador y remoto 


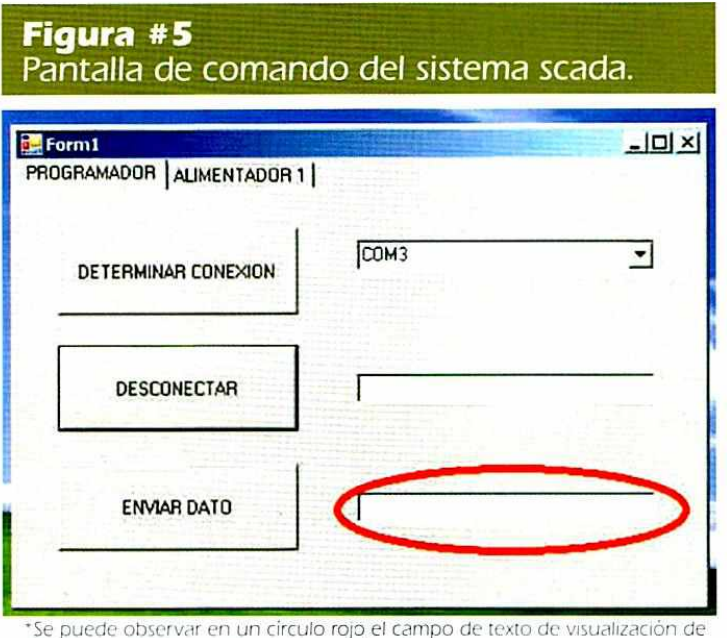

tramas receptadas por el equipo coordinador.

La pantalla de monitorización de estados (véase figura 6) informa el funcionamiento general del equipo remoto. Esta pantalla indica: (a) nivel de batería, (b) nivel del alimento en el repositorio, (c) dosis actual programada, (d) frecuencia de administración de dosis y (e) el modo de funcionamiento.

\section{Figura $\# 6$ \\ Pantalla de comando del sistema scada.}

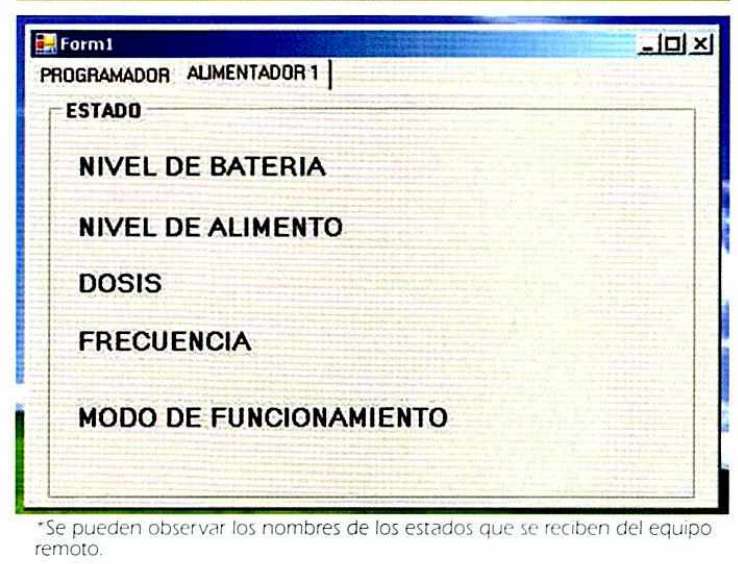

Una vez establecidas las funcionalidades para el equipo coordinador y el equipo remoto, se procedió a armar el circuito en el protoboard del sistema. Para el equipo coordinador se empleó el dispositivo que se muestra en la figura 7. Para el equipo remoto se armó el circuito en protoboard que se muestra en la figura 8 .

Utilizando este circuito se procedió a la etapa de pruebas del sistema. El programa
Figura \#7

Dispositivo interfaz entre PC y módulo Xbee.

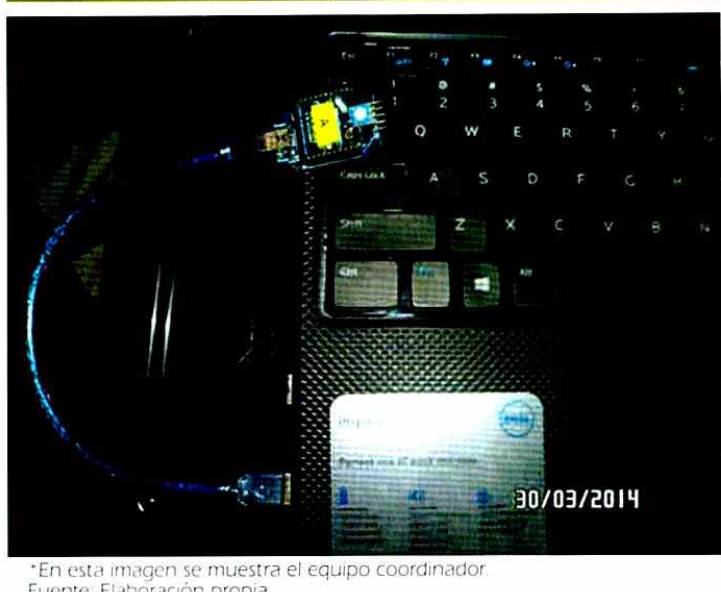

Fuente Elaboración propia.

\section{Figura \#8}

Protoboard del equipo remoto.

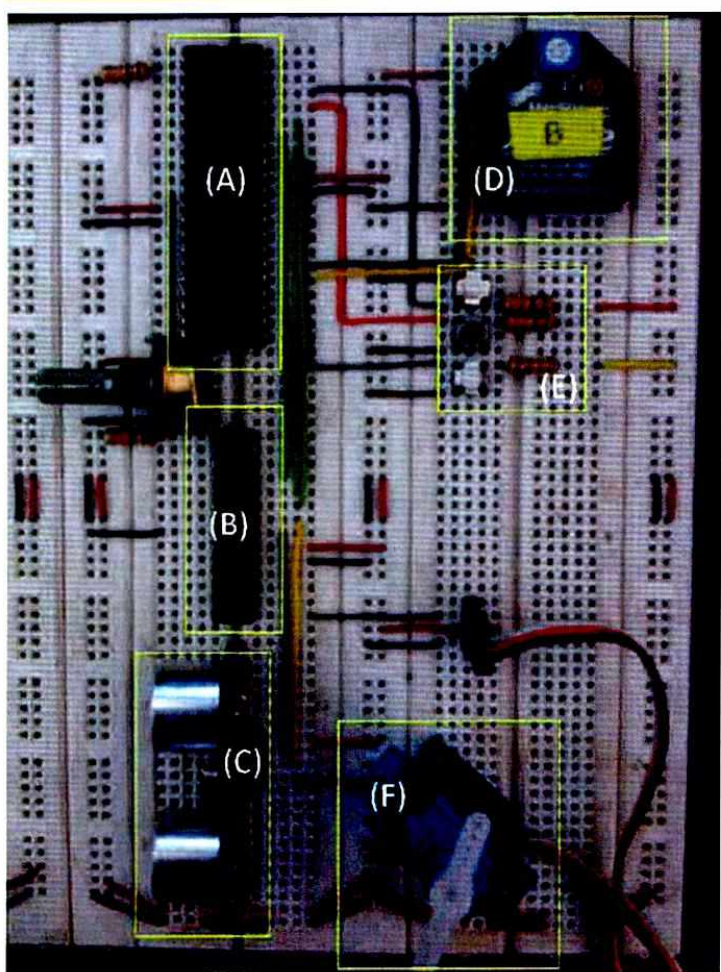

- Los equipos que se pueden apreciar son: (a) pic 16F887. (b) pic 15F886. (c) sensor ultrasónico, (d) módulo Xbee, (e) control local del equipo remoto y

Fuente: Elaboración propia.

principal que controla la comunicación serial con el módulo Xbee, el cual permite se lleve a cabo la rutina del sistema automatizado se encuentra grabado en el microcontrolador pic16F887.

$\mathrm{Al}$ igual que en los estudios como Serna (2009) o Pullés, Pino, Bonzon, y Escalona (2010), un microcontrolador de 8 bits como 
el pic16F887 era el dispositivo "eje" de los sistemas de control que en cada investigación se planteó. Esto permite disponer de un complejo organismo de supervisión para una industria a bajo costo.

En la pantalla de control se estableció la comunicación entre el equipo coordinador y el equipo remoto mediante el envío de los comandos como se muestra en la figura 9. En la figura 10 se detalla la monitorización de estados del equipo remoto en tiempo real durante la realización de las pruebas. En la figura 11 se muestra el comportamiento del módulo Xbee del equipo remoto al recibir un comando desde el equipo coordinador.

\section{Figura \# 9}

Transmisión y recepción de comandos en

pantalla de control del sistema scada.

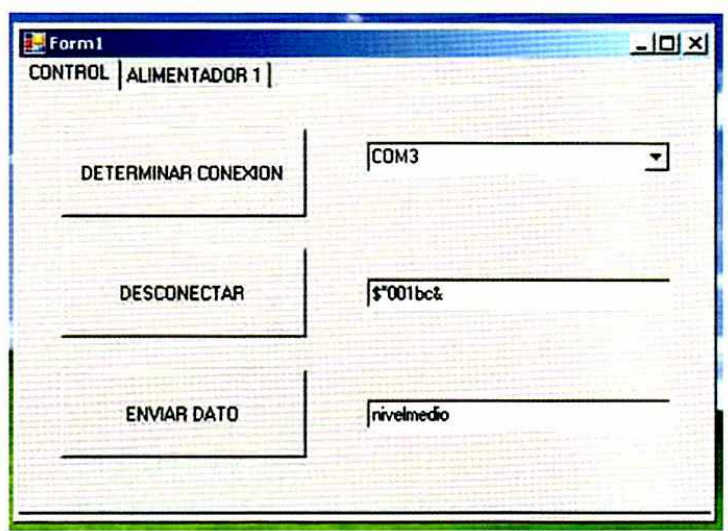

"En esta prueba se transmitió el comando " $\$$. 001 bc\&" para requerir el nive de alimento en el repositorio, para lo cual se obtuvo "nivelmedio" como Fuente Elaboración propia

\section{Figura \# 10}

Monitorización de estados del equipo

remoto en tiempo real durante la prueba.

\begin{tabular}{|c|c|}
\hline EForm1 & $-\mid[-[a|x|$ \\
\hline \multicolumn{2}{|l|}{ CONTROL ALMMENTADOR 1 | } \\
\hline NIVEL DE BATERIA & ALTO \\
\hline NIVEL DE ALIMENTO & MEDIO \\
\hline DOSIS & $\sqrt{2}$ \\
\hline FRECUENCIA & 2 \\
\hline MODO DE FUNCIONAMIENTO & AUTOMATICO \\
\hline
\end{tabular}

- Se ouede apreciar que el nivel del alimento en el repositorio es nivel la pantalla de control en la figura?

Fuente Elaboración propia.
Figura \# 11

Comunicación entre equipo coordinador y equipo remoto.

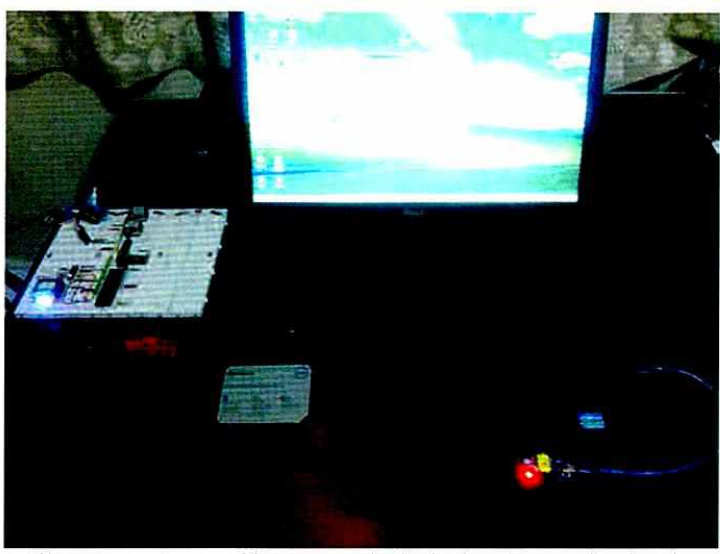

*Al momento de transmitir un comando desde el equipo coordinador, e módulo Xbee del equipo remoto muestra una luz azul intensa debido cl encendido de un led que confirma la recepción de un comando
Fuente: Elaboración propia. 


\section{Tabla \#5} Pruebas ejecutadas al sistema con los resultados deseados y los obtenidos tras
las pruebas.

\section{Prueba}

Transmisión de comando: Modificar dosis

Transmisión de comando: Modificar frecuencia

Transmisión de comando:

Requerimiento de dato de dosis

Transmisión de comando.

Requerimiento de dato de frecuencia

Transmisión de comando:

Requerimiento de dato de nivel de alimento

Transmisión de comando

Requerimiento de dato de nivel de batería

Transmisión de comando:

Requerimiento de dato de modo de funcionamiento

Funcionamiento ciclico del sistema remoto

Visualización de estados en sistema scada

Pruebas de comunicación a distancia $50 \mathrm{~m}$ con línea de vista

Pruebas de comunicación a distancia $110 \mathrm{~m}$ con línea de vista entre equipo coordinador y equipo remoto

Pruebas de comunicación a distancia $110 \mathrm{~m}$ con línea de vista entre equipo coordinador y equipo remoto, más un módulo Xbee entre estos dos últimos

Fuente: Elaboración propia.

En líneas generales los resultados obtenidos luego de ejecutadas las pruebas indican claramente que se apegaron a los resultados deseados en un principio.

El sistema mostró un funcionamiento normal, accionando el servomotor en los tiempos preprogramados y a las diferentes distancias a las que se sometió la prueba de alcance de la comunicación. Según el instructivo del módulo Xbee, la distancia máxima con visibilidad entre módulos es de $100 \mathrm{~m}$

\section{Observaciones}

Desde el sistema scada se envió el comando $\$ * 001$ aa\&. A recibir el comando el equipo remoto modifico su estado y respondió al equipo coordinador con el dato de dosis, que se modificó a "200gr".

Desde el sistema scada se envió el comando $\${ }^{\star} 001$ ab\&.Al recibir el comando el equipo remoto modificó su estado y respondió al equipo coordinador con el dato de frecuencia, que se modificó a "15min".

Desde el sistema scada se envió el comando \$*001ba\&. Al recibir el comando el equipo remoto modificó su estado y respondió al equipo coordinador con el dato de dosis, que era de "200gr".

Desde el sistema scada se envió el comando $\${ }^{\star} 001 \mathrm{bb} \&$. A recibir el comando el equipo remoto modificó su estado y respondió al equipo coordinador con el dato de frecuencia, que era "15min".

Desde el sistema scada se envió el comando $\${ }^{\star} 001 \mathrm{bc} \&$. Al recibir el comando el equipo remoto modificó su estado y respondió al equipo coordinador con el dato de nivel de alimento, que era "nivelmedio". recibir el comando el equipo remoto modificó su estado y respondió al equipo coordinador con el dato de nivel de bateria, que era "nivelalto".

Desde el sistema scada se envió el comando $\${ }^{*} 001 \mathrm{be} \&$. A recibir el comando el equipo remoto modificó su estado y respondió al equipo coordinador con el dato modo de funcionamiento, que era "automático".

El equipo remoto funcionó de forma continua durante una hora, activando cada 15 minutos el servomotor como estaba programada la rutina durante la prueba, además se enviaron tramas de requerimiento de datos desde el equipo coordinador hacia el equipo remoto y las respuestas fueron impresas en el campo de texto en el sistema scada.

El sistema scada mostró los cambios de estados esperados durante la prueba de funcionamiento continuo durante una hora.

Se enviaron tramas de requerimiento de datos de funciona miento a una distancia de $50 \mathrm{~m}$ sin ninguna novedad. La señal no se perdió y el sistema scada recibió las tramas de respuesta.

Se enviaron tramas de requerimiento de datos de funcionamiento a una distancia de $110 \mathrm{~m}$. La señal se perdió y el sistema scada no mostró las tramas de respuesta ni variaciones en la pantalla de estados.

La comunicación se retomó y el sistema scada mostró las tramas de respuesta y las variaciones esperadas en la pantalla de estados.
(Digi, 2013), y en las pruebas se pudo constatar este alcance a excepción de la prueba a $110 \mathrm{~m}$ que mediante el uso de un módulo Xbee adicional (para establecer una red tipo malla) entre el equipo remoto y el equipo coordinador se la pudo superar.

\section{Conclusiones y recomendaciones}

Realizadas las pruebas se constató que el equipo remoto es capaz de cumplir las funciones de la monitorización y control la administración de alimentos, y comunicarse 
por la red inalámbrica con el equipo coordinador de la red. Por su lado, el scada cumplió sus funciones de enrío y recepción de tramas de datos, y la correcta visualización de estados de funcionamiento del equipo remoto.

Se concluye que se cumplió con el objetivo general de esta investigación que consistía en diseñar un sistema automatizado para el control de la administración de dosis de alimentos, y que el mismo es funcional.

Se puede concluir que se cumple con los requerimientos técnicos del sistema automatizado que se aplica en la administración y' despacho de alimento en los cultivos de "tilapias".

En base a los resultados de consumo de energía de los componentes del equipo remoto, se puede concluir que se ha podido obtener un dispositivo que consume energía suficiente para ser abastecido por las baterrás $y$ sistema solar dimensionado con autonomía.

Gracias al diseño flexible de las parámetros de alimentación en los equipos remotos se recomienda el uso de este dispositivo en otro tipos de cultivos como camarones, truchas, chame, y demás cultivos afines.

\section{Bibliografia}

Buratti, C., Martaló, M., Verdone, R., \& Ferrari, G. (2011). Sensor Networks with IEEE 802.15.4 Systems. Springer: Springer-Verlag Berlin Heiderlberg.

Chia-Ming, W., Ruay-Shiung, C., Pu-I, L., \& Jei-Hsiang, Y. (30 de septiembre de 2011). An innovative scheme for increasing connectivityand life of ZigBee networks. The Journal of Supercomputing, 136-140.

Chowdhury, D. (2011). Optimal feeding rate for nile "tilapias" (Oreochromis niloti- cus). Department of Animal and Aquacultural Sciences.

Digi. (2013). Digi Your M2M Expert. Recuperado el 20 de octubre de 2013, de http:// www.digi.com/technology/rf-articles/ wireless-zigbee

Digi International Inc. (2012). Product Manual 17.xEx - 802.15.4 Protocol. Minnetonka: 2012 Digi International, Inc.

Draghuciu, N., \& Marcel Perte, V. 5.-5. (2012). Applications of Microcontrollers in Automobiles Field. Journal of Electrical \& Eilectronics Engineering, 53-56.

Farahani, S. (2008). ZigBee Wireless Networks and Transcievers. Jordan Hill: Elsevier.

Gislason, D. (2008). ZigBee Wireless Netmorking. Burlington: Elsevier.

IEEE. (1 de octubre de 2003). Part 15.4: Wireless Medium Access Control (MAC) and Physical Layer (PHY) Specifications for Low-Rate Wireless Personal Area Networks (LR-WPANs). New York City, New York, Estados Unidos.

Lakhoua, M., \& Laadhari, H. (2013). Supervision of the Natural Gas Station Using a SCADA System. Jorrnal of Electrical d Electronics Engineering, 51-54.

Magdy, M., \& Mohamed, A. (2008, vol. 20, $\left.\mathrm{n}^{\circ} 3\right)$. Relationship between dietary protein source and feeding frequency during feeding nile "tilapias", Oreochromis niloticus (L.) cultured in Concrete Tanks. Journal of Applied Aquaculture, 200 - 212.

Martínez, C., Chávez, M., \& Olvera, M. (no viembre de 1989). La nutricion $y^{\prime}$ alimentación en la acuicultura de América Latina. Una diagnosis. Recuperado el 2 de Noviembre 
de 2013, de Depósito de documentos de la FAO: http://www.fao.org/docrep/ field/003/AB459S/AB459S00.htm

Molish, A. (2011). Wireless Communications. Chichester: John Wiley \& Sons Ltd.

Organización de las Naciones Unidas para la Alimentación y la Agricultura. (2008). "tilapias" del Nilo - Métodos de suministro de alimentos. Recuperado el 2 de noviembre de 2013, de Sistema de Información sobre Alimentos y Recursos Fertilizantes para la Acuicultura: http://www.fao.org/ fishery/affris/perfiles-de-las-especies/ nile-"tilapias"/metodos-de-suministrode-alimentos/es/

Organización de las Uniones Unidas para la Alimentacióny la Agricultura. (16 de noviembre de 2006). Métodos sencillos para la acuicultura. Recuperado el 3 de noviembre de 2013, de 10 Nutrición y alimentación de los peces: ftp://ftp.fao.org/fi/ CDrom/FAO_Training/FAO_Training/ General/x6709s/x6709s10.htm

Pullés, J., Pino, A., Bonzon, J., \& Escalona, O. (2010). Desarrollo de aplicaciones de monitorización zigbee para sistemas de calentamiento de agua con energía solar. Ciencia en su PC, 41-57.

Romo, J., \& Alvarez, M. (3 de marzo de 2009). Repositorio de la Escuela Superior Politecnica del Litoral. Recuperado el 20 de noviembre de 2013, de Evaluación técnica y económica del uso de bandejas en la alimentación de piscinas camaroneras: http://www.dspace.espol.edu.ec/bitstream/123456789/1756/1/3492.pdf

Serna, S. (2009). Especificación de perfil zigbee para monitorización y control de plantas industriales. Revista Tecnológicas, 167-185.
Sheikh, A. (2004). Wireless Comunications Theory and Techniques. Norwell: Kluwer Academic Publishers Group.

Singal, T. (2010). Wireless Communications. Nueva Delhi: Tata McGraw Hill.

\section{Gustavo Delgado Cortez}

Ing. electrónico de la Universidad Espíritu Santo - UEES

E-mail: gudelgado@uees.edu.ec

\section{Pablo Jácome Rulz}

Ingeniero en Electrónica y Telecomunicaciones de la Escuela Superior Politécnica Del Litoral (ESPOL)

Realizando estudios para la obtención de título Magister en Ciencias de la Ingeniería, mención Ingeniería Eléctrica en la Universidad de Chile

Universidad Espíritu Santo - Ecuador

E-mail:psjacome@gmail.com 